\title{
FINITE DIFFERENCES AND BOUNDARY ELEMENT METHODS FOR NON-STATIONARY VISCOUS INCOMPRESSIBLE FLOW
}

\author{
WERNER VARNHORN \\ Technical University of Dresden, Institute of Numerical Mathematics \\ 01062 Dresden, Germany
}

\begin{abstract}
We consider an implicit fractional step procedure for the time discretization of the non-stationary Stokes equations in smoothly bounded domains of $\mathbb{R}^{3}$. We prove optimal convergence properties uniformly in time in a scale of Sobolev spaces, under a certain regularity of the solution. We develop a representation for the solution of the discretized equations in the form of potentials and the uniquely determined solution of some system of boundary integral equations. For the numerical computation of the potentials and the solution of the boundary integral equations a boundary element method of collocation type is carried out.
\end{abstract}

1. Introduction and notation. Let $T>0$ be given and $G \subset \mathbb{R}^{3}$ be a bounded domain with a sufficiently smooth compact boundary $S$. In $(0, T) \times G$ we consider the non-stationary Stokes equations

$$
\begin{gathered}
D_{t} v-\nu \Delta v+\nabla p=F, \quad \operatorname{div} v=0, \\
v_{\mid S}=0, \quad v_{\mid t=0}=v_{0} .
\end{gathered}
$$

These equations describe the linearized motion of a viscous incompressible fluid: the vector $v=\left(v_{1}(t, x), v_{2}(t, x), v_{3}(t, x)\right)$ represents the velocity field and the scalar $p=p(t, x)$ the kinematic pressure function of the fluid at time $t \in(0, T)$ and at position $x \in G$. The constant $\nu>0$ is the kinematic viscosity, and the external force density $F$ together with the initial velocity $v_{0}$ are the given data. The condition $\operatorname{div} v=0$ means the incompressibility of the fluid, and $v=0$ on the boundary $S$ expresses the no-slip condition, i.e. the fluid adheres to the boundary.

1991 Mathematics Subject Classification: 31B10, 35K50, 39A11, 45B05, 65(M06, N38, R20), $76 \mathrm{D} 05$.

The paper is in final form and no version of it will be published elsewhere. 
It is the aim of the present paper to develop a method for the numerical solution of (1.1). This method consists of three steps. In the first step, finite differences in time are used in order to transform (1.1) into certain boundary value problems. In the second step, these boundary value problems are studied with methods of potential theory. This leads to a representation of their solutions consisting of volume and surface potentials, whose densities have to be determined from a system of boundary integral equations. Thirdly, for the discretization of the boundary integral equations and the numerical computation of the potentials a boundary element method of collocation type and suitable quadrature methods are used.

Let us consider the following semidiscrete first order approximation scheme for the Stokes equations (1.1): Setting

$$
h=T / N>0, \quad t_{k}=k \cdot h \quad(k=0,1, \ldots, N),
$$

we approximate the solution $v, p$ of (1.1) at time $t_{k}$ by the solution $v^{k}, p^{k}(k=$ $1,2, \ldots, N)$ of the following equations in $G$ :

$$
\begin{gathered}
\left(v^{k}-v^{k-1}\right) h^{-1}-\nu \Delta v^{k}+\nabla p^{k}=h^{-1} \int_{(k-1) h}^{k h} F(t) d t, \\
\operatorname{div} v^{k}=0, \quad v^{0}=v_{0}, \quad v^{k}{ }_{\mid S}=0 .
\end{gathered}
$$

Here $F$ and $v_{0}$ are the given data. Thus for every $k=1,2,3, \ldots, N$ we have to determine in $G$ the solution $v^{k}, q^{k}$ of the boundary value problem

$$
\begin{gathered}
(\lambda-\Delta) v^{k}+\nabla q^{k}=F^{\lambda, k-1} \quad\left(\lambda=(\nu h)^{-1}>0\right), \\
\operatorname{div} v^{k}=0, \quad v^{k}{ }_{\mid S}=0
\end{gathered}
$$

where $q^{k}=p^{k} / \nu$ and

$$
F^{\lambda, k-1}(x)=\lambda\left(v^{k-1}(x)+\int_{(k-1) h}^{k h} F(t, x) d t\right) .
$$

Using methods of potential theory, we find a representation of the solution $v^{k}, q^{k}$ in the form

$$
\left(v^{k}(x), q^{k}(x)\right)=\left(V_{\lambda} F^{\lambda, k-1}\right)(x)+\left(D_{\lambda} \Psi\right)(x), \quad x \in G .
$$

Here $V_{\lambda} F^{\lambda, k-1}$ is a hydrodynamic volume potential with density $F^{\lambda, k-1}$, and $D_{\lambda} \Psi$ is a double layer potential, whose unknown source density $\Psi$ has to be determined from the boundary integral equations

$$
-\left(V_{\lambda}^{*} F^{\lambda, k-1}\right)(x)=\frac{1}{2} \Psi(x)+\left(D_{\lambda}^{*} \Psi\right)(x)-\left(P_{N} \Psi\right)(x), \quad x \in S .
$$

Here the superscript $*$ refers to the velocity part of the above potentials, $\left(D_{\lambda}^{*} \Psi\right)$ is the direct value of the hydrodynamic double layer potential for the velocity, and $P_{N} \Psi$ is a one-dimensional perturbation operator, which ensures that the solution $\Psi$ is unique in the space of continuous vector fields on $S$ (compare [1]). For the 
spatial discretization of (1.6) we use a boundary element method of collocation type as described in $[6,17]$.

At this point, let us introduce our notations. Throughout the paper, $G \subset \mathbb{R}^{3}$ is a bounded domain having a compact boundary $S$ of class $C^{2}$. In the following, all functions are real valued. As usual, $C_{0}^{\infty}(G)$ denotes the space of smooth functions defined in $G$ with compact support, and $L^{2}(G)$ is the Lebesgue (Hilbert) space equipped with the scalar product and norm

$$
\langle f, g\rangle=\int_{G} f(x) g(x) d x, \quad\|f\|=\langle f, f\rangle^{1 / 2},
$$

respectively. For functions $f, g \in L^{2}(G)$ we need the following well-known relations:

$$
\begin{aligned}
\langle f-g, f+g\rangle & =\|f\|^{2}-\|g\|^{2}, \\
\langle f-g, 2 f\rangle & =\|f\|^{2}-\|g\|^{2}+\|f-g\|^{2}, \\
2\langle f, g\rangle & \leq 2\|f\|\|g\| \leq\|f\|^{2}+\|g\|^{2} .
\end{aligned}
$$

The Sobolev (Hilbert) space $H^{m}(G)(m \in \mathbb{N}=\{0,1,2, \ldots\})$ is the space of functions $f$ such that $D^{\alpha} f \in L^{2}(G)$ for all $\alpha=\left(\alpha_{1}, \alpha_{2}, \alpha_{3}\right) \in \mathbb{N}^{3}$ with $|\alpha|=$ $\alpha_{1}+\alpha_{2}+\alpha_{3} \leq m$. Its norm is denoted by

$$
\|f\|_{m}=\|f\|_{H^{m}(G)}=\left(\sum_{|\alpha| \leq m}\left\|D^{\alpha} f\right\|^{2}\right)^{1 / 2}, \quad D^{\alpha}=D_{1}^{\alpha_{1}} D_{2}^{\alpha_{2}} D_{3}^{\alpha_{3}},
$$

where $D_{k}=d / d x_{k}(k=1,2,3)$ is the distributional derivative. The completion of $C_{0}^{\infty}(G)$ with respect to $\|\cdot\|_{m}$ is denoted by

$$
H_{0}^{m}(G) \quad\left(H_{0}^{0}(G)=H^{0}(G)=L^{2}(G)\right) .
$$

If $f \in H_{0}^{1}(G)$, in particular, we have Poincaré's inequality

$$
\|f\|^{2} \leq C_{G}\|\nabla f\|^{2},
$$

where the constant $\lambda_{1}=C_{G}^{-1}$ is the smallest eigenvalue of the Laplace operator $-\Delta$ in $G$ with zero boundary condition [10, p. 11].

The spaces $C_{0}^{\infty}(G)^{3}, L^{2}(G)^{3}, H^{m}(G)^{3}, \ldots$ are the corresponding spaces of vector fields $u=\left(u_{1}, u_{2}, u_{3}\right)$. Here the norm and scalar product are denoted as in the scalar case, i.e. for example

$$
\langle u, v\rangle=\sum_{k=1}^{3}\left\langle u_{k}, v_{k}\right\rangle, \quad\|u\|=\langle u, u\rangle^{1 / 2}=\left(\int_{G}|u(x)|^{2} d x\right)^{1 / 2},
$$

where $|u(x)|=\left(u_{1}(x)^{2}+u_{2}(x)^{2}+u_{3}(x)^{2}\right)^{1 / 2}$ is the Euclidian norm of $u(x) \in \mathbb{R}^{3}$. The completions of

$$
C_{0, \sigma}^{\infty}(G)^{3}=\left\{u \in C_{0}^{\infty}(G)^{3} \mid \operatorname{div} u=0\right\}
$$


with respect to the norms $\|\cdot\|$ and $\|\cdot\|_{1}$ are important spaces for the treatment of the Stokes equations. They are denoted by

$$
\mathcal{H}(G)^{3}, \quad \mathcal{V}(G)^{3},
$$

respectively. In $H_{0}^{1}(G)^{3}$ and $\mathcal{V}(G)^{3}$ we also use

$$
\langle\nabla u, \nabla v\rangle=\sum_{k, j=1}^{3}\left\langle D_{k} u_{j}, D_{k} v_{j}\right\rangle, \quad\|\nabla u\|=\langle\nabla u, \nabla u\rangle^{1 / 2}
$$

as scalar product and norm $[16$, p. 5]. Moreover, we need the $B$-valued spaces $C^{m}(J, B)$ and $H^{m}(a, b, B), m \in \mathbb{N}$, where $J \subset \mathbb{R}$ is a compact interval, where $a, b \in \mathbb{R}(a<b)$, and where $B$ is any of the spaces above. In case of $C^{0}($,$) we$ simply write $C\left(\right.$, ), and we use $H, V, H^{m}, \ldots$ instead of $H(G), V(G), H^{m}(G), \ldots$ if the domain of definition is clear from the context. Finally, let

$$
P: L^{2}(G)^{3} \rightarrow \mathcal{H}(G)^{3}
$$

denote the orthogonal projection (see $[16$, p. 15]). Then we have

$$
L^{2}(G)^{3}=\mathcal{H}(G)^{3} \oplus\left\{v \in L^{2}(G)^{3} \mid v=\nabla p \text { for some } p \in H^{1}(G)\right\},
$$

which means

$$
\langle u, \nabla p\rangle=0 \quad \text { for all } u \in \mathcal{V}(G)^{3} \text { and } p \in H^{1}(G) .
$$

2. Discretization of time. Because the projection $P$ from (1.9) commutes with the strong time derivative $D_{t}$, from the Stokes equations (1.1) we obtain the following evolution equations for the function $t \rightarrow v(t) \in \mathcal{H}(G)^{3}$ :

$$
D_{t} v(t)-\nu P \Delta v(t)=P F(t) \quad(t \in(0, T)), \quad v(0)=v_{0} .
$$

In this case, the condition $\operatorname{div} v=0$ and the boundary condition $v=0$ on $S$ are satisfied in the sense that we require $v(t) \in \mathcal{V}(G)^{3}$ for all $t \in(0, T)$.

Concerning the solvability of the evolution equations (2.1), it is known (see $[10$, p. 89]) that for

$$
v_{0} \in H^{2}(G)^{3} \cap \mathcal{V}(G)^{3}, \quad F \in H^{1}\left(0, T, \mathcal{H}(G)^{3}\right),
$$

there is a unique solution $v$ of (2.1) in $G$ such that

$$
\begin{aligned}
v & \in C\left([0, T], H^{2}(G)^{3} \cap \mathcal{V}(G)^{3}\right), \\
D_{t} v & \in C\left([0, T], \mathcal{H}(G)^{3}\right) \cap L^{2}\left(0, T, H^{1}(G)^{3}\right),
\end{aligned}
$$

and that there is some constant $K$ depending only on $G, \nu, F, v_{0}$ and not on $t \in[0, T]$ such that for all $t \in[0, T]$

$$
\int_{0}^{t}\left\|\nabla D_{\sigma} v(\sigma)\right\|^{2} d \sigma \leq K, \quad\|v(t)\|_{2} \leq K, \quad\left\|D_{t} v(t)\right\| \leq K .
$$


Let us now consider the discretized equations (1.2) under the weaker assumptions

$$
v_{0} \in \mathcal{H}(G)^{3}, \quad F \in L^{2}\left(0, T, \mathcal{H}(G)^{3}\right) .
$$

Using $P$ as above and noting that $F=P F$ we obtain in $G(h=T / N>0)$,

$$
\left(v^{k}-v^{k-1}\right)-h \nu P \Delta v^{k}=\int_{(k-1) h}^{k h} F(t) d t, \quad v^{0}=v_{0} .
$$

It is known that under the above assumptions (2.5) there is a unique solution

$$
v^{k} \in H^{2}(G)^{3} \cap \mathcal{V}(G)^{3} \quad(k=1,2, \ldots, N)
$$

of (2.6): If we define the Stokes operator $A$ to be Friedrichs' extension of $-P \Delta$ in $\mathcal{H}(G)^{3}$, then its domain of definition $D(A)$ is $H^{2}(G)^{3} \cap \mathcal{V}(G)^{3}$ ([2], see also $\left[5\right.$, p. 270]). Because $\lambda=(\nu h)^{-1}>0$ belongs to the resolvent set of $-A$, the equations

$$
v^{k}=(\lambda+A)^{-1} F^{\lambda, k-1}, \quad F^{\lambda, k-1} \in \mathcal{H}(G)^{3}
$$

(see (1.4)) are (successively for $k=1,2, \ldots, N)$ uniquely solvable with $v^{k} \in$ $H^{2}(G)^{3} \cap \mathcal{V}(G)^{3}$, as asserted.

To prove the convergence of the discretized equations (2.6) to the evolution equations (2.1) and to estimate the discretization error, we use the Lax-Richtmyer approach "stability + consistency $\rightarrow$ convergence", originally developed in [11]. Let us define

$$
\begin{aligned}
\left(\Pi_{h} v\right)\left(t_{k}\right) & =v\left(t_{k}\right)-v\left(t_{k-1}\right)-\nu h P \Delta v\left(t_{k}\right), \\
\left(\Pi_{h}\left\{v^{j}\right\}\right)\left(t_{k}\right) & =v^{k}-v^{k-1}-\nu h P \Delta v^{k} .
\end{aligned}
$$

Then the defect

$$
e^{k}=v^{k}-v\left(t_{k}\right)
$$

satisfies the identity

$$
e^{k}-e^{k-1}-\nu h P \Delta e^{k}=\left(\Pi_{h}\left\{v^{j}\right\}\right)\left(t_{k}\right)-\left(\Pi_{h} v\right)\left(t_{k}\right)=R^{k},
$$

which is used to obtain estimates of $e^{k}$ in terms of the right hand side $R^{k}$ $(\approx$ Stability). Then the behaviour of

$$
\begin{aligned}
R^{k} & =\int_{(k-1) h}^{k h}\left(D_{t} v(t)-\nu P \Delta v(t)\right) d t-\left\{\left(v\left(t_{k}\right)-v\left(t_{k-1}\right)\right)-h \nu P \Delta v\left(t_{k}\right)\right\} \\
& =\int_{(k-1) h}^{k h}-\nu P \Delta\left(v(t)-v\left(t_{k}\right)\right) d t=-\nu P \Delta E^{k}
\end{aligned}
$$

as $h$ tends to zero ( $\approx$ Consistency) follows from the regularity properties of the exact solution of the Stokes equations (2.1). 
Theorem. Let $T>0, N \in \mathbb{N}$, and $G \subset \mathbb{R}^{3}$ be a bounded domain with a smooth boundary $S$ of class $C^{2}$. Assuming $(2.2)$, let $v$ and $v^{k}(k=1,2, \ldots, N)$ denote the solution of (2.1) and (2.6), respectively. Then the discretization error $e^{k}$ (see (2.8)) satisfies the following estimates:

$$
\begin{array}{r}
\left\|e^{k}\right\|^{2}+\sum_{j=1}^{k}\left(h \nu\left\|\nabla e^{j}\right\|^{2}+\left\|e^{j}-e^{j-1}\right\|^{2}\right) \leq K h^{2}, \\
\left\|\nabla e^{k}\right\|^{2}+\sum_{j=1}^{k}\left(2(h \nu)^{-1}\left\|e^{j}-e^{j-1}\right\|^{2}+\frac{1}{2}\left\|\nabla\left(e^{j}-e^{j-1}\right)\right\|^{2}\right) \leq K h .
\end{array}
$$

Here the constant $K$ depends only on $G, \nu$, and the data. Moreover, we even have convergence with respect to the $H^{2}$-norm:

$$
\max \left\{\left\|e^{k}\right\|_{2} \mid k=1,2, \ldots, N\right\}=o(1) \quad \text { as } h \rightarrow 0 \text { or } N \rightarrow \infty .
$$

Proof. From (2.9) and (2.10) we obtain for the defect $e^{k}$ the identity

$$
\left(e^{k}-e^{k-1}\right)-h \nu P \Delta e^{k}=-\nu P \Delta E^{k} .
$$

Multiplying (2.11) scalarly in $L^{2}$ by $2 e^{k}$ and using (1.7), we obtain

$$
\begin{aligned}
\left\|e^{k}\right\|^{2}-\left\|e^{k-1}\right\|^{2}+\left\|e^{k}-e^{k-1}\right\|^{2}+ & 2 h \nu\left\|\nabla e^{k}\right\|^{2}=2 \nu\left\langle\nabla E^{k}, \nabla e^{k}\right\rangle \\
& \leq 2 \cdot(h \nu)^{1 / 2}\left\|\nabla e^{k}\right\| \cdot\left(h^{-1} \nu\right)^{1 / 2}\left\|\nabla E^{k}\right\| \\
& \leq h \nu\left\|\nabla e^{k}\right\|^{2}+h^{-1} \nu\left\|\nabla E^{k}\right\|^{2}=S_{1}+S_{2} .
\end{aligned}
$$

Because of

$$
\begin{aligned}
S_{2} & =h^{-1} \nu\left\|\int_{(k-1) h}^{k h} \int_{t}^{k h} D_{\sigma} \nabla v(\sigma) d \sigma d t\right\|^{2} \leq \nu \int_{(k-1) h}^{k h}\left\|\int_{(k-1) h}^{k h}\left|D_{\sigma} \nabla v(\sigma)\right| d \sigma\right\|^{2} d t \\
& \leq \nu h\left\|\int_{(k-1) h}^{k h}|\nabla v(\sigma)| d \sigma\right\|^{2} \leq \nu h^{2} \int_{(k-1) h}^{k h}\left\|D_{\sigma} \nabla v(\sigma)\right\|^{2} d \sigma,
\end{aligned}
$$

we find

$$
\begin{aligned}
\left\|e^{k}\right\|^{2}-\left\|e^{k-1}\right\|^{2}+\left\|e^{k}-e^{k-1}\right\|^{2}+h \nu\left\|\nabla e^{k}\right\|^{2} & \\
& \leq \nu h^{2} \int_{(k-1) h}^{k h}\left\|D_{\sigma} \nabla v(\sigma)\right\|^{2} d \sigma
\end{aligned}
$$

for all $k=1,2, \ldots, N$. Thus, using $\left\|e^{0}\right\|^{2}=0$ and (2.4), the first estimate is proved.

Next let us multiply (2.11) scalarly in $L^{2}$ by $2\left(e^{k}-e^{k-1}\right)$. We obtain

$$
\begin{aligned}
2 \| e^{k}-e^{k-1} & \|^{2}+2 h \nu\left\langle\nabla e^{k}, \nabla\left(e^{k}-e^{k-1}\right)\right\rangle \\
& =2\left\|e^{k}-e^{k-1}\right\|^{2}+h \nu\left(\left\|\nabla e^{k}\right\|^{2}-\left\|\nabla e^{k-1}\right\|^{2}+\left\|\nabla\left(e^{k}-e^{k-1}\right)\right\|^{2}\right) \\
& =2 \nu\left\langle\nabla E^{k}, \nabla\left(e^{k}-e^{k-1}\right)\right\rangle
\end{aligned}
$$




$$
\begin{aligned}
& \leq 2 \cdot(h \nu / 2)^{1 / 2}\left\|\nabla\left(e^{k}-e^{k-1}\right)\right\| \cdot\left(2 h^{-1} \nu\right)^{1 / 2}\left\|\nabla E^{k}\right\| \\
& \leq(h \nu / 2)\left\|\nabla\left(e^{k}-e^{k-1}\right)\right\|^{2}+2 h^{-1} \nu\left\|\nabla E^{k}\right\|^{2}=S_{3}+2 S_{2} .
\end{aligned}
$$

Using the above estimate for $S_{2}$ again, we have

$$
\begin{aligned}
2\left\|e^{k}-e^{k-1}\right\|^{2}+h \nu\left(\left\|\nabla e^{k}\right\|^{2}-\left\|\nabla e^{k-1}\right\|^{2}\right)+ & (h \nu / 2)\left\|\nabla\left(e^{k}-e^{k-1}\right)\right\|^{2} \\
& \leq 2 \nu h^{2} \int_{(k-1) h}^{k h}\left\|D_{\sigma} \nabla v(\sigma)\right\|^{2} d \sigma,
\end{aligned}
$$

hence

$$
\begin{aligned}
\left\|\nabla e^{k}\right\|^{2}-\left\|\nabla e^{k-1}\right\|^{2}+2(h \nu)^{-1}\left\|e^{k}-e^{k-1}\right\|^{2}+ & \frac{1}{2}\left\|\nabla\left(e^{k}-e^{k-1}\right)\right\|^{2} \\
& \leq 2 h \int_{(k-1) h}^{k h}\left\|D_{\sigma} \nabla v(\sigma)\right\|^{2} d \sigma,
\end{aligned}
$$

which implies the second estimate.

Next we want to prove convergence with respect to the $H^{2}$-norm. From (2.11) we conclude

which implies

$$
P \Delta e^{k}=(h \nu)^{-1}\left(e^{k}-e^{k-1}\right)+h^{-1} P \Delta E^{k},
$$

$$
\left\|P \Delta e^{k}\right\|^{2} \leq 2(h \nu)^{-2}\left\|e^{k}-e^{k-1}\right\|^{2}+2 h^{-2}\left\|P \Delta E^{k}\right\|^{2} .
$$

By (2.3) we find the following estimate for the second term:

$$
\begin{aligned}
2 h^{-2}\left\|P \Delta E^{k}\right\|^{2} & \leq 2 h^{-2}\left\|\int_{(k-1) h}^{k h} P \Delta\left(v(t)-v\left(t_{k}\right)\right) d t\right\|^{2} \\
& \leq 2 \max _{\substack{\sigma, \tau \in[0, T] \\
|\sigma-\tau| \leq h}}\|P \Delta(v(\sigma)-v(\tau))\|^{2}=o(1) \quad \text { as } h \rightarrow 0 .
\end{aligned}
$$

It remains to show that also the first term of (2.13) tends to zero. Using

$$
T^{k}=\left(e^{k}-e^{k-1}\right) / h \quad(k=1,2, \ldots, N)
$$

for abbreviation, form (2.11) we obtain the identity

$$
\begin{aligned}
& T^{k}-T^{k-1}-h \nu P \Delta T^{k} \\
& \quad=-h^{-1} \nu P \Delta\left\{\int_{(k-1) h}^{k h}\left(v(t)-v\left(t_{k}\right)\right) d t-\int_{(k-2) h}^{(k-1) h}\left(v(t)-v\left(t_{k-1}\right)\right) d t\right\} \\
& =-h^{-1} \nu P \Delta G^{k},
\end{aligned}
$$

where $G^{k}$ is defined by the above term in braces. Scalar multiplication in $L^{2}$ by $2 T^{k}$ yields as above

$$
\begin{aligned}
\left\|T^{k}\right\|^{2}-\left\|T^{k-1}\right\|^{2}+\left\|T^{k}-T^{k-1}\right\|^{2}+ & 2 h \nu\left\|\nabla T^{k}\right\|^{2} \\
& \leq h \nu\left\|\nabla T^{k}\right\|^{2}+h^{-3} \nu\left\|\nabla G^{k}\right\|^{2}
\end{aligned}
$$


hence

$$
\left\|T^{k}\right\|^{2}-\left\|T^{k-1}\right\|^{2}+\left\|T^{k}-T^{k-1}\right\|^{2}+h \nu\left\|\nabla T^{k}\right\|^{2} \leq h^{-3} \nu\left\|\nabla G^{k}\right\|^{2} .
$$

Because

$$
G^{k}=-\int_{(k-1) h}^{k h} \int_{t}^{k h}\left(D_{\sigma} v(\sigma)-D_{\sigma} v(\sigma-h)\right) d \sigma d t,
$$

we find the estimate

$$
\left\|\nabla G^{k}\right\|^{2} \leq h^{3} \int_{(k-1) h}^{k h}\left\|D_{\sigma} \nabla(v(\sigma)-v(\sigma-h))\right\|^{2} d \sigma .
$$

Thus from (2.14) we obtain

$$
\begin{aligned}
\left\|T^{k}\right\|^{2}-\left\|T^{k-1}\right\|^{2}+\left\|T^{k}-T^{k-1}\right\|^{2}+ & h \nu\left\|\nabla T^{k}\right\|^{2} \\
& \leq \nu \int_{(k-1) h}^{k h}\left\|D_{t} \nabla(v(t)-v(t-h))\right\|^{2} d t,
\end{aligned}
$$

and

$$
\begin{aligned}
\left\|T^{k}\right\|^{2} & +\sum_{j=2}^{k}\left(\left\|T^{j}-T^{j-1}\right\|^{2}+\nu h\left\|\nabla T^{j}\right\|^{2}\right) \\
& \leq\left\|T^{1}\right\|^{2}+\nu \int_{h}^{T}\left\|D_{t} \nabla(v(t)-v(t-h))\right\|^{2} d t=o(1) \quad \text { as } h \rightarrow 0,
\end{aligned}
$$

because the integral vanishes as $h \rightarrow 0$, and because by $(2.12)$ (note $\left\|e^{0}\right\|=0$ ),

$$
\left\|T^{1}\right\|^{2}=\left\|\left(e^{1}-e^{0}\right) h^{-1}\right\|^{2} \leq \nu \int_{0}^{h}\left\|D_{t} \nabla v(t)\right\|^{2} d t=o(1) .
$$

Thus (2.15) implies that also the first term of (2.13) tends to zero as $h \rightarrow 0$, hence $\left\|P \Delta e^{k}\right\|^{2}=o(1)$ as $h \rightarrow 0$, and the asserted convergence with respect to the $H^{2}$-norm follows by means of Cattabriga's estimate [2]. This proves the theorem.

3. Potential theory. Because every time step $t_{k}=k h(k=1,2, \ldots, N \in$ $\mathbb{N} ; h=T / N>0$ ) requires the solution of the boundary value problem (1.3), we consider for fixed $h, k$, and $\lambda=(h \nu)^{-1}>0$ in $G$ the system

$$
(\lambda-\Delta) u+\nabla q=F \quad(\lambda>0), \quad \operatorname{div} u=0, \quad u_{\mid S}=0 .
$$

Let us define the formal differential operator of (3.1) by

$$
S_{\lambda}:\left(\begin{array}{l}
u \\
q
\end{array}\right) \rightarrow S_{\lambda}^{u}=\left(\begin{array}{c}
(\lambda-\Delta) u+\nabla q \\
\nabla \cdot u
\end{array}\right)
$$


and let

$$
S_{\lambda}^{\prime}:\left(\begin{array}{l}
u \\
q
\end{array}\right) \rightarrow S_{\lambda}^{\prime}{ }_{q}^{u}=\left(\begin{array}{c}
(\lambda-\Delta) u-\nabla q \\
-\nabla \cdot u
\end{array}\right)
$$

denote its formally adjoint operator. To construct an explicit solution $u, q$ of (3.1) with methods of potential theory, we first need the singular fundamental tensor $E_{\lambda}=\left(E_{j k}^{\lambda}\right)_{j, k=1, \ldots, 4}$, i.e. a solution of $S_{\lambda} E_{\lambda}=\delta \mathrm{I}_{4}$ in the space of tempered distributions. Here $\delta$ is Dirac's distribution in $\mathbb{R}^{3}, \mathrm{I}_{4}$ the $4 \times 4$ unity matrix, and $S_{\lambda} E_{\lambda}=\left(S E_{1}^{\lambda}, S E_{2}^{\lambda}, S E_{3}^{\lambda}, S E_{4}^{\lambda}\right)$ with columns $E_{k}^{\lambda}=\left(E_{j k}^{\lambda}\right)_{j=1, \ldots, 4}$ for $k=1, \ldots, 4$. To compute $E=E_{\lambda}$ we use Fourier transformation. Setting

$$
E_{j k}^{*}(\alpha)=\left(F E_{j k}\right)(\alpha)=c_{\pi} \int_{\mathbb{R}^{3}} \exp (-i \alpha \cdot x) E_{j k}(x) d x
$$

with $c_{\pi}=(2 \pi)^{-3 / 2}$ we obtain for every $k=1, \ldots, 4$ the vector identity (in the distributional sense)

$$
\left(\begin{array}{c}
\left(|\alpha|^{2} E_{j k}^{*}+\lambda E_{j k}^{*}+i \alpha_{j} E_{4 k}^{*}\right)_{j=1,2,3} \\
i \sum_{j=1}^{3} \alpha_{j} E_{j k}^{*}
\end{array}\right)(\alpha)=c_{\pi}\left(\delta_{j k}\right)_{j=1, \ldots, 4} .
$$

Here $\delta_{j k}$ denotes the Kronecker symbol. From these identities the Fourier transform $E^{*}$ of $E$ can be easily determined: For $j, k=1,2,3$ we have

$$
\begin{aligned}
& E_{j k}^{*}(\alpha)=\delta_{j k} c_{\pi}\left(|\alpha|^{2}+\lambda\right)^{-1}-\alpha_{j} \alpha_{k} c_{\pi}|\alpha|^{-2}\left(|\alpha|^{2}+\lambda\right)^{-1}, \\
& E_{4 k}^{*}(\alpha)=E_{k 4}^{*}(\alpha)=-i \alpha_{k} c_{\pi}|\alpha|^{-2}, \\
& E_{44}^{*}(\alpha)=c_{\pi}\left(1+\lambda|\alpha|^{-2}\right) .
\end{aligned}
$$

To compute $E$ from $E^{*}$ by inverse Fourier transformation, let

$$
E_{j k}(x)=\left(F^{-1} E_{j k}^{*}\right)(x)=c_{\pi} \int_{\mathbb{R}^{3}} \exp (i \alpha \cdot x) E_{j k}^{*}(\alpha) d \alpha .
$$

Then we obtain for $j, k=1,2,3$,

$$
\begin{gathered}
E_{j k}=\delta_{j k} F^{-1}\left(c_{\pi} /\left(|\alpha|^{2}+\lambda\right)\right)+D_{j k}^{2} F^{-1}\left(c_{\pi}|\alpha|^{-2}\left(|\alpha|^{2}+\lambda\right)^{-1}\right), \\
E_{4 k}=E_{k 4}=D_{k} F^{-1}\left(c_{\pi} /|\alpha|^{2}\right), \quad E_{44}=F^{-1} c_{\pi}+\lambda F^{-1}\left(c_{\pi} /|\alpha|^{2}\right),
\end{gathered}
$$

with

$$
\begin{gathered}
\left(F^{-1} c_{\pi}\right)(x)=\delta(x), \quad\left(F^{-1}\left(c_{\pi} /|\alpha|^{2}\right)\right)(x)=(4 \pi|x|)^{-1}, \\
\left(F^{-1}\left(c_{\pi} /\left(|\alpha|^{2}+\lambda\right)\right)\right)(x)=(4 \pi|x|)^{-1} \exp (-\sqrt{\lambda}|x|), \\
\left(F^{-1}\left(c_{\pi}|\alpha|^{-2}\left(|\alpha|^{2}+\lambda\right)^{-1}\right)\right)(x)=(4 \pi \lambda|x|)^{-1}(1-\exp (-\sqrt{\lambda}|x|)) .
\end{gathered}
$$

Note that the right hand sides of the last three equations are fundamental solutions of the operators $-\Delta,-\Delta+\lambda$, and $(-\Delta+\lambda)(-\Delta)$, respectively [12]. Now some elementary calculations yield (compare also [13, p. 206]) that the funda- 
mental tensor $E_{\lambda}=\left(E_{j k}^{\lambda}(x)\right)_{j, k=1, \ldots, 4}$ has the following form:

$$
\begin{gathered}
E_{j k}^{\lambda}(x)=\frac{1}{4 \pi}\left\{\frac{\delta_{j k}}{|x|} e_{1}(-\sqrt{\lambda}|x|)+\frac{x_{j} x_{k}}{|x|^{3}} e_{2}(-\sqrt{\lambda}|x|)\right\} \quad(k, j \neq 4), \\
e_{1}(\varepsilon)=\sum_{n=0}^{\infty} \frac{(n+1)^{2}}{(n+2) !} \varepsilon^{n}=\exp (\varepsilon)\left(1-\varepsilon^{-1}+\varepsilon^{-2}\right)-\varepsilon^{-2} \\
e_{2}(\varepsilon)=\sum_{n=0}^{\infty} \frac{1-n^{2}}{(n+2) !} \varepsilon^{n}=\exp (\varepsilon)\left(-1+3 \varepsilon^{-1}-3 \varepsilon^{-2}\right)+3 \varepsilon^{-2}, \\
E_{4 k}^{\lambda}(x)=E_{k 4}^{\lambda}(x)=\frac{x_{k}}{4 \pi|x|^{3}} \quad(k \neq 4), \quad E_{44}^{\lambda}(x)=\delta(x)+\frac{\lambda}{4 \pi|x|} .
\end{gathered}
$$

Using the exponential representation of the functions $e_{1}, e_{2}$ we immediately obtain the behaviour of $E_{\lambda}(x)$ for $x \rightarrow 0$ and $x \rightarrow \infty$. Setting $r=|x|$ we have for $j, k \neq 4$ :

$$
\begin{array}{ll}
E_{j k}^{\lambda}(x)=O\left(r^{-1}\right) & \text { as } r \rightarrow 0, \\
E_{j k}^{\lambda}(x)=O\left(r^{-3}\right) & \text { as } r \rightarrow \infty(\lambda>0), \\
E_{4 k}^{\lambda}(x)=O\left(r^{-2}\right) & \text { as } r \rightarrow 0 \text { or } r \rightarrow \infty .
\end{array}
$$

Note that $E_{j k}^{\lambda}(\lambda>0)$ decays stronger than $E_{j k}^{0}(j, k \neq 4)$ as $r \rightarrow \infty$.

Now using the right hand side $F$ from (3.1) and the fundamental tensor $E_{\lambda}$, we can construct the hydrodynamical volume potential

$$
(U(x), Q(x))=\int_{G}\left\langle\left(\begin{array}{c}
F(y) \\
0
\end{array}\right), E_{\lambda}(x-y)\right\rangle d y,
$$

which satisfies the equations $S_{\lambda}{ }_{Q}^{U}=\left(\begin{array}{c}F \\ 0\end{array}\right)$ in $G$ due to its construction. Here and in the sequel, for $\xi \in \mathbb{R}^{n}$ and matrices $A=\left(A_{j i}\right) \in \mathbb{R}^{n} \times \mathbb{R}^{m}(n, m \in \mathbb{N})$ we use

$$
\langle\xi, A\rangle=\left(\sum_{j=1}^{n} \xi_{j} A_{j 1}, \ldots, \sum_{j=1}^{n} \xi_{j} A_{j m}\right),
$$

obtaining a row with $m$ components.

In order to represent the solution of $\left(S_{\lambda}\right)$ by means of potentials we need the hydrodynamical Green's formulae. They are given in terms of the formal differential operators

$$
S_{\lambda}:\left(\begin{array}{l}
u \\
q
\end{array}\right) \rightarrow S_{\lambda} \begin{aligned}
& u \\
& q
\end{aligned}, \quad S_{\lambda}^{\prime}:\left(\begin{array}{l}
u \\
q
\end{array}\right) \rightarrow S_{\lambda}^{\prime}{ }_{q}^{u}
$$

from above, and their corresponding adjoint stress tensors, which are defined by

$$
\begin{gathered}
T:\left(\begin{array}{l}
u \\
q
\end{array}\right) \rightarrow T_{q}^{u}=\left(-\nabla u-(\nabla u)^{\mathrm{T}}+q \mathrm{I}_{3}\right), \\
T^{\prime}:\left(\begin{array}{l}
u \\
q
\end{array}\right) \rightarrow T^{\prime}{ }_{q}^{u}=\left(-\nabla u-(\nabla u)^{\mathrm{T}}-q \mathrm{I}_{3}\right) .
\end{gathered}
$$


Here $(\nabla u)^{\mathrm{T}}$ is the transposed matrix of $\nabla u=\left(D_{i} u_{k}\right)_{k, i=1,2,3}$ and $\mathrm{I}_{3}$ the $3 \times 3$ unit matrix.

Let us assume that $u, v \in C^{2}(G)^{3} \cap C^{1}(\bar{G})^{3}$ are divergence-free vector fields, that $q, p \in C^{1}(G) \cap C^{0}(\bar{G})$, and that $S_{\lambda_{q}}^{u}, S_{\lambda p}^{\prime v} \in L^{1}(G)^{3}(\lambda>0)$. Then we have Green's first identity

$$
\begin{aligned}
& \int_{G}\left\langle S_{\lambda}{ }_{q}^{u},\left(\begin{array}{l}
v \\
p
\end{array}\right)\right\rangle d y \\
= & \int_{S}\left\langle T_{q}^{u} N, v\right\rangle d o_{y}+\int_{G}\langle\lambda u, v\rangle d y+\int_{G} \frac{1}{2}\left\langle\nabla u+(\nabla u)^{\mathrm{T}}, \nabla v+(\nabla v)^{\mathrm{T}}\right\rangle d y,
\end{aligned}
$$

and Green's second identity

$$
\begin{aligned}
& \int_{G}\left\{\left\langle S_{\lambda}^{u},\left(\begin{array}{l}
v \\
p
\end{array}\right)\right\rangle-\left\langle\left(\begin{array}{l}
u \\
q
\end{array}\right), S_{\lambda}^{\prime} \begin{array}{l}
v \\
p
\end{array}\right)\right\} d y \\
& =\int_{S}\left\{\left\langle T_{q}^{u} N, v\right\rangle-\left\langle u, T^{\prime}{ }_{p}^{v} N\right\rangle\right\} d o_{y} .
\end{aligned}
$$

Here we use $\langle\xi, \eta\rangle=\sum_{k=1}^{n} \xi_{k} \eta_{k}$ for $\xi, \eta \in \mathbb{R}^{n}$ and $\langle A, B\rangle=\sum_{i, k=1}^{n} A_{i k} B_{i k}$ for matrices $A, B \in \mathbb{R}^{n} \times \mathbb{R}^{n}(n \in \mathbb{N})$. The vector $N=N(y) \in \mathbb{R}^{3}$ denotes the exterior normal at $y \in S$ and $T_{q}^{u} N$ indicates the usual matrix vector product.

Now applying Green's second identity with a solution $u \in C^{2}(G)^{3} \cap C^{1}(\bar{G})^{3}$, $q \in C^{1}(G) \cap C^{0}(\bar{G})$ of $S_{\lambda_{q}^{u}}^{u}=\left(\begin{array}{c}F \\ 0\end{array}\right)$, and with $v, p$ being the columns of the fundamental tensor $E_{\lambda}$, by cutting off the singularity in $x \in G$ we obtain the following representation (compare [14, p. 335]) of $u$ and $q$ in $x \in G$ ( $N$ denotes the exterior normal to the $C^{2}$-boundary $\left.S\right)$ :

$$
\begin{aligned}
\int_{G}\left\langle\left(\begin{array}{c}
F(y) \\
0
\end{array}\right), E_{\lambda}(x-y)\right\rangle d y- & (u(x), q(x)) \\
= & \int_{S}\left\langle T_{q}^{u}(y) N(y), E_{\lambda}^{(\mathrm{r})}(x-y)\right\rangle d o_{y} \\
& -\int_{S}\left\langle u(y), T_{y}^{\prime} E_{\lambda}(x-y) N(y)\right\rangle d o_{y} .
\end{aligned}
$$

Here $E_{\lambda}^{(\mathrm{r})}$ is the $3 \times 4$ matrix obtained from $E_{\lambda}$ by eliminating the last row, and the product in the last boundary integral equation is defined as follows: Treating the 4 columns of $E_{\lambda}$ with $T^{\prime}$ yields four $3 \times 3$ matrices, which, multiplied by $N$, give four columns with 3 components, hence a $3 \times 4$ matrix. The subscript $y$ in $T_{y}^{\prime}$ means differentiation with respect to $y$.

The representation formula (3.7) suggests introducing hydrodynamical boundary layer potentials for general vector source densities $\Psi=\left(\Psi_{1}, \Psi_{2}, \Psi_{3}\right) \in C(S)^{3}$. 
For $x \in \mathbb{R}^{3} \backslash S$ we define the single layer potential

$$
\left(E_{\lambda} \Psi\right)(x)=\int_{S}\left\langle\Psi(y), E_{\lambda}^{(\mathrm{r})}(x-y)\right\rangle d o_{y}
$$

and the double layer potential

$$
\left(D_{\lambda} \Psi\right)(x)=\int_{S}\left\langle\Psi(y), T_{y}^{\prime} E_{\lambda}(x-y) N(y)\right\rangle d o_{y}
$$

Because $E_{\lambda}=E_{\lambda}^{\mathrm{T}}$, the single layer potential can be represented by

$$
\left(E_{\lambda} \Psi\right)(x)=\int_{S} E_{\lambda}^{(\mathrm{c})}(x-y) \Psi(y) d o_{y}
$$

Here the $4 \times 3$ matrix $E_{\lambda}^{(\mathrm{c})}$ is obtained from $E_{\lambda}$ by eliminating the last column and $E_{\lambda}^{(\mathrm{c})} \Psi$ indicates the usual matrix vector product. If no confusion is possible, row representation and column representation will be identified. In order to develop a similar representation for the double layer potential we proceed as follows. Due to $D_{y_{i}} E_{j k}^{\lambda}(x-y)=-D_{x_{i}} E_{j k}^{\lambda}(x-y)(i, j=1,2,3 ; k=1, \ldots, 4)$ and recalling the definition of $T$ and $T^{\prime}$ we have $T_{y}^{\prime} E_{k}^{\lambda}(x-y)=-T_{x} E_{k}^{\lambda}(x-y)$ where $E_{k}^{\lambda}$ denotes the $k$ th column of $E_{\lambda}(k=1, \ldots, 4)$. Defining the $3 \times 4$ matrix $\left(D_{\lambda}(x, y)\right)^{\mathrm{T}}=$ $-T_{x} E_{\lambda}(x-y) N(y)$, we first obtain the row vector

$$
\left(D_{\lambda} \Psi\right)(x)=\int_{S}\left\langle\Psi(y),\left(D_{\lambda}(x, y)\right)^{\mathrm{T}}\right\rangle d o_{y}
$$

and then the column

$$
\left(D_{\lambda} \Psi\right)(x)=\int_{S} D_{\lambda}(x, y) \Psi(y) d o_{y}
$$

where the $4 \times 3$ matrix $D_{\lambda}(x, y)$ is defined by

$$
D_{\lambda}(x, y)=\left(-T_{x} E_{\lambda}(x-y) N(y)\right)^{\mathrm{T}}=\left(\left(-T_{x} E_{k}^{\lambda}(x-y)\right)_{i j} N_{j}(y)\right)_{k i} .
$$

Both the single layer potential (3.8) and the double layer potential (3.9) are analytic functions in $\mathbb{R}^{3} \backslash S$ and satisfy there the homogeneous differential equations

$$
S_{\lambda}{ }_{q}^{u}=\left(\begin{array}{l}
0 \\
0
\end{array}\right) .
$$

By elementary calculations we find (compare [13, p. 210]) that the $4 \times 3$ kernel matrix $D_{\lambda}=\left(D_{k i}^{\lambda}(x, y)\right)_{k=1, \ldots, 4 ; i=1,2,3}$ of the double layer potential $D_{\lambda} \Psi$ has the 
following form: Setting $r=x-y$ and $N=N(y)$ we have

$$
\begin{aligned}
D_{k i}^{\lambda}(x, y)= & -\frac{1}{4 \pi}\left\{\frac{r_{k} N_{i}}{|r|^{3}} d_{1}(-\sqrt{\lambda}|r|)\right. \\
& -\left(\frac{N_{k} r_{i}}{|r|^{3}}+\delta_{k i} \frac{r \cdot N}{|r|^{3}}\right) d_{2}(-\sqrt{\lambda}|r|) \\
& \left.+\frac{r_{k} r_{i} r \cdot N}{|r|^{5}}\left(3-3 d_{1}(-\sqrt{\lambda}|r|)+2 d_{2}(-\sqrt{\lambda}|r|)\right)\right\}, \\
d_{1}(\varepsilon)= & \sum_{n=2}^{\infty} \frac{2\left(n^{2}-1\right)}{(n+2) !} \varepsilon^{n}=\exp (\varepsilon)\left(2-6 \varepsilon^{-1}+6 \varepsilon^{-2}\right)-6 \varepsilon^{-2}+1, \\
d_{2}(\varepsilon)= & \sum_{n=2}^{\infty} \frac{n\left(n^{2}-1\right)}{(n+2) !} \varepsilon^{n}=\exp (\varepsilon)\left(\varepsilon-3+6 \varepsilon^{-1}-6 \varepsilon^{-2}\right)+6 \varepsilon^{-2}, \\
D_{4 i}^{\lambda}(x, y)= & -\frac{1}{4 \pi}\left\{6 \frac{r_{i} r \cdot N}{|r|^{5}}+\frac{\lambda N_{i}}{|r|}-2 \frac{N_{i}}{|r|^{3}}\right\}-N_{i} \delta(r) .
\end{aligned}
$$

The series representation above yields $d_{1}(0)=d_{2}(0)=0$, hence as $\lambda \rightarrow 0$ we obtain from (3.6) the well known (see [10, p. 55], [14, p. 336]) double layer kernel matrix for the Stokes equations $\left(S_{0}\right)$ :

$$
\begin{aligned}
& D_{k i}^{0}(x, y)=-\frac{3}{4 \pi} \frac{r_{k} r_{i} r \cdot N}{|r|^{5}} \quad(k, i=1,2,3), \\
& D_{4 i}^{0}(x, y)=-\frac{1}{2 \pi}\left(\frac{3 r \cdot N r_{i}}{|r|^{5}}-\frac{N_{i}}{|r|^{3}}\right)-N_{i} \delta(r) \quad(i=1,2,3) .
\end{aligned}
$$

It follows easily that the last summand in $d_{1}$ comes from the pressure $q$. This term determines the decay for $\varrho=|r|=|x-y| \rightarrow 0$ and $\varrho \rightarrow \infty$. Hence for $k, i \neq 4$ we have $(\lambda>0)$

$$
\begin{aligned}
D_{k i}^{\lambda}(x, y)=O\left(\varrho^{-2}\right) & \text { as } \varrho \rightarrow 0 \text { or } \varrho \rightarrow \infty(\lambda>0), \\
D_{4, i}^{\lambda}(x, y)=O\left(\varrho^{-3}\right) & \text { as } \varrho \rightarrow 0, \\
D_{4 i}^{\lambda}(x, y)=O\left(\varrho^{-1}\right) & \text { as } \varrho \rightarrow \infty .
\end{aligned}
$$

In the following we consider the normal stresses of the single layer potential $E_{\lambda} \Psi$, which are defined in a neighbourhood $U \subseteq \mathbb{R}^{3}$ of $S$ for $x \in U \backslash S$ and $\Psi \in C(S)^{3}$ by

$$
\left(H_{\lambda}^{*} \Psi\right)(x)=\int_{S} T_{x}\left(E_{\lambda}^{(\mathrm{c})}(x-y) \Psi(y)\right) N(\widetilde{x}) d o_{y} .
$$

Here the superscript $*$ indicates a column vector with 3 components, and $N(\widetilde{x})$ denotes the outward unit normal at $\widetilde{x} \in S$, where $\widetilde{x}$ is the unique projection of $x \in U \backslash S$ on $S$. Note that $S \in C^{2}$ allows the construction of parallel surfaces $[15, \S 200]$, which implies the existence of such a neighbourhood $U$. If we use the 
representation

$$
\left(H_{\lambda}^{*} \Psi\right)(x)=\int_{S} H_{\lambda}(x, y) \Psi(y) d o_{y}
$$

with some $3 \times 3$ matrix $H_{\lambda}(x, y)$, then for $x, y \in S$ we have

$$
H_{\lambda}(x, y)=D_{\lambda}^{(\mathrm{r})}(y, x)^{\mathrm{T}}
$$

with $D_{\lambda}^{(\mathrm{r})}$ obtained by eliminating the last row of the $4 \times 3$ matrix $D_{\lambda}$ given above.

The next statements concern the continuity properties of the potentials, if $x \in \mathbb{R}^{3} \backslash S$ approaches a point $z \in S$. For $x \in \mathbb{R}^{3} \backslash S$ let

$$
\begin{aligned}
& \left(E_{\lambda}^{*} \Psi\right)(x)=\int_{S} E_{\lambda}^{(\mathrm{r}, \mathrm{c})}(x-y) \Psi(y) d o_{y}, \\
& \left(D_{\lambda}^{*} \Psi\right)(x)=\int_{S} D_{\lambda}^{(\mathrm{r})}(x, y) \Psi(y) d o_{y}
\end{aligned}
$$

denote the single layer and the double layer potential corresponding to the velocity part of the potentials, respectively. Here $E_{\lambda}^{(\mathrm{r}, \mathrm{c})}$ is the $3 \times 3$ matrix obtained from $E_{\lambda}$ by eliminating the last row $(\approx \mathrm{r})$ and the last column $(\approx \mathrm{c})$. We first consider some potentials with special densities.

It is well known (see $[10$, p. 56], $[14$, p. 337]) that for the case $\lambda=0$ we have

$$
\left(D_{0}^{*} \beta\right)(x)=\int_{S} D_{0}(x, y) \beta d o_{y}= \begin{cases}\beta, & x \in G, \\ \frac{1}{2} \beta, & x \in S, \\ 0, & x \in \mathbb{R}^{3} \backslash \bar{G},\end{cases}
$$

where $D_{0}$ is the $3 \times 3$ matrix defined in (3.11) and $\beta \in \mathbb{R}^{3}$ is a constant column vector. For $\lambda>0$, however,

$$
\left(D_{\lambda}^{*} \beta\right)(x)+\lambda \int_{G} E_{\lambda}^{(\mathrm{r}, \mathrm{c})}(x-y) \beta d y= \begin{cases}\beta, & x \in G, \\ \frac{1}{2} \beta, & x \in S, \\ 0, & x \in \mathbb{R}^{3} \backslash \bar{G} .\end{cases}
$$

Moreover, if $N$ denotes the outward unit normal field on $S$, then for the single layer potential $E_{\lambda} N(\lambda>0)$ with density $N$ we have

$$
\left(E_{\lambda} N\right)(x)=\int_{S} E_{\lambda}^{(\mathrm{c})}(x-y) N(y) d o_{y}= \begin{cases}-\left(\begin{array}{l}
0 \\
1
\end{array}\right), & x \in G, \\
-\frac{1}{2}\left(\begin{array}{l}
0 \\
1
\end{array}\right), & x \in S, \\
\left(\begin{array}{l}
0 \\
0
\end{array}\right), & x \in \mathbb{R}^{3} \backslash \bar{G},\end{cases}
$$

which follows from Green's second identity, and implies $\left(E_{\lambda}^{*} N\right)(x)=0$ for all $x \in \mathbb{R}^{3}$.

Next let us study the continuity properties of potentials with general continuous source densities. Setting

$$
w^{\mathrm{i}}(z)=\lim _{\substack{x \rightarrow z \in S \\ x \in G}} w(x), \quad w^{\mathrm{e}}(z)=\lim _{\substack{x \rightarrow z \in S \\ x \in \mathbb{R}^{3} \backslash \bar{G}}} w(x)
$$


we obtain on the boundary $S$ the important relations

$$
\begin{aligned}
\left(E_{\lambda}^{*} \Psi\right)^{\mathrm{i}} & =E_{\lambda}^{*} \Psi=\left(E_{\lambda}^{*} \Psi\right)^{\mathrm{e}}, \\
\left(D_{\lambda}^{*} \Psi\right)^{\mathrm{i}}-D_{\lambda}^{*} \Psi & =\frac{1}{2} \Psi=D_{\lambda}^{*} \Psi-\left(D_{\lambda}^{*} \Psi\right)^{\mathrm{e}}, \\
\left(H_{\lambda}^{*} \Psi\right)^{\mathrm{e}}-H_{\lambda}^{*} \Psi & =\frac{1}{2} \Psi=H_{\lambda}^{*} \Psi-\left(H_{\lambda}^{*} \Psi\right)^{\mathrm{i}},
\end{aligned}
$$

where $E_{\lambda}^{*} \Psi, D_{\lambda}^{*} \Psi$, and $H_{\lambda}^{*} \Psi$ are defined by (3.14), (3.15), and (3.13), respectively.

Now let $G^{\mathrm{c}}=\mathbb{R}^{3} \backslash \bar{G}$ be the complementing exterior domain having the same boundary $S$ as $G$. We consider the following boundary value problem: For a given boundary value $b \in C(S)^{3}$ find $u \in C^{2}(G)^{3} \cap C(\bar{G})^{3}, q \in C^{1}(G) \cap C(\bar{G})$ satisfying

$$
S_{\lambda}{ }_{q}^{u}=\left(\begin{array}{l}
0 \\
0
\end{array}\right) \quad \text { in } G, \quad u=b \quad \text { on } S \text {. }
$$

We refer to this problem as to the interior hydrodynamic Dirichlet problem. Besides (3.22) we also consider the exterior hydrodynamic Neumann problem

$$
S_{\lambda}{ }_{q}^{u}=\left(\begin{array}{l}
0 \\
0
\end{array}\right) \quad \text { in } G^{\mathrm{c}}, \quad T_{q}^{u} N=b \quad \text { on } S,
$$

which is adjoint to (3.22). Using Green's first identity we can easily prove that regular solutions $u, q$ of the exterior Neumann problem are uniquely determined provided that we require

$$
u(x)=O\left(r^{-2}\right), \quad \nabla u(x)=O\left(r^{-1}\right), \quad q(x)=O\left(r^{-1}\right)
$$

as $r=|x| \rightarrow \infty(\lambda>0)$, a condition which takes into account the special decay properties of the potentials (compare (3.3) and (3.12)).

Concerning the interior Dirichlet problem, $u$ is uniquely determined, while $q$ is uniquely determined up to an additive constant only.

In the following we prove the existence of a solution $u, q$ of the interior Dirichlet problem using the method of boundary integral equations. Let $b \in C(S)^{3}$ be given with

$$
\int_{S} b \cdot N d o=0
$$

Choosing at $x \in G$ the ansatz $\left(\begin{array}{l}u \\ q\end{array}\right)(x)=\left(D_{\lambda} \Psi\right)(x)$ as double layer potential, due to the jump relations we obtain on $S$ the weakly singular ( $S$ is of class $C^{2}$ ) boundary integral equations

$$
b=\frac{1}{2} \Psi+\left(D_{\lambda}^{*} \Psi\right) \quad \text { on } S,
$$

which is a Fredholm system of the second kind on $C(S)^{3}$. To solve it we have to consider the corresponding homogeneous adjoint system

$$
0=\frac{1}{2} \Phi+\left(H_{\lambda}^{*} \Phi\right) \quad \text { on } S .
$$

It follows from (3.18) that the normal vector $N \in C(S)^{3}$ is a solution of (3.27): Due to $\left(H_{\lambda}^{*} N\right)(x)=\left(T\left(E_{\lambda} N\right)\right)(x) N(\widetilde{x})=-N(\widetilde{x})$ if $x \in G$ (for $\widetilde{x}$ see above (3.13)) 
and $\left(H_{\lambda}^{*} N\right)(x)=0$ if $x \in G^{\mathrm{c}}$, from (3.21) we obtain

$$
0=\frac{1}{2} N+\left(H_{\lambda}^{*} N\right) \quad \text { on } S .
$$

Moreover, if $\Phi \in C(S)^{3}$ is any solution of (3.27), then we have $\Phi=\beta N$ with some constant $\beta \in \mathbb{R}$. To see this, consider the single layer potential $\left(\begin{array}{l}u \\ q\end{array}\right)=E_{\lambda} \Phi$ defined in (3.8). It decays as required in (3.24), and it solves the exterior Neumann problem (3.23) with zero boundary data due to (3.21) and (3.27). Thus we have $E_{\lambda} \Phi=\left(\begin{array}{l}0 \\ 0\end{array}\right)$ in $G^{\mathrm{c}}$ from the uniqueness statement, and $E_{\lambda}^{*} \Phi=0$ on $S$ using (3.19). This again implies that $E_{\lambda} \Phi$ also solves the interior Dirichlet problem with zero boundary data, and the corresponding uniqueness statement yields $E_{\lambda} \Phi=\left(\begin{array}{l}0 \\ \alpha\end{array}\right)$ in $G$, with some constant $\alpha \in \mathbb{R}$. Because $H_{\lambda}^{*} \Phi=0$ in $G^{\text {c }}$ and $H_{\lambda}^{*} \Phi=\alpha N$ in $G$, the assertion follows by (3.21). Now using well known facts of Fredholm's theory on integral equations of the second kind in spaces of continuous functions it follows that the condition (3.25) is necessary and sufficient for the existence of a solution $\Psi \in C(S)^{3}$ of $(3.26)$.

Because (3.27) has a unique nontrivial solution $\Phi=N$, the homogeneous version of (3.26) has a nontrivial solution, too. For numerical purposes, however, it is desirable to deal with uniquely solvable systems. Following [1], this can be achieved as follows: Instead of (3.26) consider the system of boundary integral equations

$$
b=\frac{1}{2} \Psi+\left(D_{\lambda}^{*} \Psi\right)-\left(P_{N} \Psi\right) \quad \text { on } S
$$

with the one-dimensional operator $P_{N}: C(S)^{3} \rightarrow C(S)^{3}$ given by

$$
\left(P_{N} \Psi\right)(x)=N(x) \int_{S} N \cdot \Psi d o .
$$

Because the normal field $N$ forms a basis of the nullspace of the operator $\frac{1}{2} \mathrm{I}_{3}+H_{\lambda}^{*}$, which is adjoint to $\frac{1}{2} \mathrm{I}_{3}+D_{\lambda}^{*}$, the system (3.28) is uniquely solvable in $C(S)^{3}$ [1], and moreover, in case of (3.25) its solution $\Psi$ solves (3.26), too. The latter follows easily by multiplying (3.28) with $N$, integrating over $S$, and noting that

$$
\int_{S}\left(D_{\lambda}^{*} \Psi\right) \cdot N d o=\int_{S} \Psi \cdot\left(H_{\lambda}^{*} N\right) d o=-\frac{1}{2} \int_{S} \Psi \cdot N d o .
$$

Thus we have shown

Theorem. Let $b \in C(S)^{3}$ with (3.25) be given on a $C^{2}$-boundary $S$ of a bounded domain $G \subseteq \mathbb{R}^{3}$, and let $0<\lambda \in \mathbb{R}$. Then the interior hydrodynamic Dirichlet problem (3.22) has a solution $u \in C^{2}(G)^{3} \cap C(\bar{G})^{3}, q \in C^{1}(G)$. Here $u$ is uniquely determined, while $q$ is unique up to an additive constant only. The solution $u, q$ can be represented in $G$ as a pure double layer potential $\left(\begin{array}{l}u \\ q\end{array}\right)(x)=$ $\left(D_{\lambda} \Psi\right)(x)$, where the source density $\Psi \in C(S)^{3}$ is the unique solution of the second kind Fredholm system of boundary integral equations

$$
b=\frac{1}{2} \Psi+\left(D_{\lambda}^{*} \Psi\right)-\left(P_{N} \Psi\right) \quad \text { on } S .
$$


Here $D_{\lambda}^{*} \Psi$ is the velocity part of $D_{\lambda} \Psi$ and $P_{N}: C(S)^{3} \rightarrow C(S)^{3}$ is defined by $\left(P_{N} \Psi\right)(x)=N(x) \int_{S} N \cdot \Psi d o$.

4. Spatial discretization. Summarizing the results from the last two sections we find that the potential representation given in (1.5) defines an approximate solution $\left(v^{k}(x), q^{k}(x)\right)$ of the Stokes equations (1.1) at time $t_{k}=k h(k=$ $1,2, \ldots, N)$. It depends on the solution $\Psi$ of the system (1.6) of boundary integral equations, which - for each time step - has the form (3.28). For the discretization of (3.28) we choose a collocation procedure developed in [6] (see also [17]). To be concrete, in the following let us restrict our considerations to the case of the unit ball $G \subseteq \mathbb{R}^{3}$ with the boundary $S$ and let us use the parametrization

$$
f: S^{\wedge}=[0,1]^{2} \rightarrow S, \quad f(\vartheta, \eta)=\left(x_{1}, x_{2}, x_{3}\right) \in S,
$$

i.e. $x_{1}=\sin (\pi \vartheta) \cos (2 \pi \eta), x_{2}=\sin (\pi \vartheta) \sin (2 \pi \eta), x_{3}=\cos (\pi \vartheta)$. For the sake of illustration, in the following we suppress some analytical problems due to the non-uniqueness of the inverse mapping $f^{-1}$. For $L \in \mathbb{N}$ let $\sigma=(2 L)^{-1}$ and define on $S^{\wedge}$ the so-called collocation grid

$$
C_{\sigma}^{\wedge}=\left\{x^{\wedge}=(i \sigma, j \sigma) \mid i, j=0, \ldots, 2 L\right\}
$$

consisting of $(2 L+1)^{2}$ collocation points, and the integration grid

$$
J_{\sigma}^{\wedge}=\left\{y^{\wedge}=((i+0.5) \sigma,(j+0.5) \sigma) \mid i, j=0, \ldots, 2 L-1\right\}
$$

consisting of $(2 L)^{2}$ integration points. For $y^{\wedge}=((i+0.5) \sigma,(j+0.5) \sigma) \in J_{\sigma}^{\wedge}$ let

$$
Q_{y}^{\wedge}=\{(\vartheta, \eta) \mid i \sigma<\vartheta<(i+1) \sigma, j \sigma<\eta<(j+1) \sigma\}
$$

be the square with length $\sigma$ and center $y^{\wedge}$. The projections of these sets on $S$ are denoted by

Setting

$$
C_{\sigma}=f\left(C_{\sigma}^{\wedge}\right), \quad J_{\sigma}=f\left(J_{\sigma}^{\wedge}\right), \quad Q_{y}=f\left(Q_{y}^{\wedge}\right)
$$

$$
\omega(\tau)= \begin{cases}\tau+1 & \text { for }-1 \leq \tau \leq 0 \\ 1-\tau & \text { for } 0 \leq \tau \leq 1 \\ 0 & \text { elsewhere }\end{cases}
$$

for every $x^{\wedge}=\left(x_{1}, x_{2}\right) \in C_{\sigma}^{\wedge}$ let us define a bi-linear $B$-spline

$$
\xi^{\wedge}: S^{\wedge} \rightarrow \mathbb{R}, \quad \xi^{\wedge}(\vartheta, \eta)=\omega\left(\left(\vartheta-x_{1}\right) / \sigma\right) \omega\left(\left(\eta-x_{2}\right) / \sigma\right) .
$$

These splines are used for interpolation: the interpolate $P_{\sigma}^{\wedge} \Phi^{\wedge}: S^{\wedge} \rightarrow \mathbb{R}^{3}$ of some vector function $\Phi^{\wedge}: S^{\wedge} \rightarrow \mathbb{R}^{3}$ is defined by

$$
\left(P_{\sigma}^{\wedge} \Phi^{\wedge}\right)(\vartheta, \eta)=\sum_{x^{\wedge} \in C_{\sigma}^{\wedge}} \Phi^{\wedge}\left(x^{\wedge}\right) \xi^{\wedge}(\vartheta, \eta),
$$

and we have $\left(P_{\sigma}^{\wedge} \Phi^{\wedge}\right)\left(z^{\wedge}\right)=\Phi^{\wedge}\left(z^{\wedge}\right)$ for all $z^{\wedge} \in C_{\sigma}^{\wedge}$. Analogously, we call

$$
P_{\sigma} \Phi=\left(P_{\sigma}^{\wedge}(\Phi \circ f)\right) \circ f^{-1}
$$

the interpolate of $\Phi: S \rightarrow \mathbb{R}^{3}$. 
Let us now go back to the system (3.28) of boundary integral equations and look for an approximate solution

$$
\Psi_{\sigma}=\Psi_{\sigma}^{\wedge} \circ f^{-1}
$$

where the vector function $\Psi_{\sigma}^{\wedge}: S^{\wedge} \rightarrow \mathbb{R}^{3}$ has the form

$$
\Psi_{\sigma}^{\wedge}(\vartheta, \eta)=\sum_{x^{\wedge} \in C_{\sigma}^{\wedge}} \alpha\left(x^{\wedge}, \sigma\right) \xi^{\wedge}(\vartheta, \eta)
$$

Here the unknown coefficients $\alpha\left(x^{\wedge}, \sigma\right) \in \mathbb{R}^{3}$ have to be determined from the collocation procedure

$$
P_{\sigma} b=P_{\sigma}\left(\frac{1}{2} \Psi_{\sigma}+\left(D_{\lambda, \sigma}^{*} \Psi_{\sigma}\right)-\left(P_{N, \sigma} \Psi_{\sigma}\right)\right),
$$

where (compare (3.15) and (3.28))

$$
\begin{aligned}
& \left(D_{\lambda, \sigma}^{*} \Psi_{\sigma}\right)(x)=\sum_{y \in J_{\sigma}} D_{\lambda}^{(\mathrm{r})}(x, y) \Phi_{\sigma}(y)\left|Q_{y}\right| \quad\left(x \notin J_{\sigma}\right), \\
& \left(P_{N, \sigma} \Psi_{\sigma}\right)(x)=N(x) \sum_{y \in J_{\sigma}} N(y) \cdot \Phi_{\sigma}(y)\left|Q_{y}\right| \quad(x \in S),
\end{aligned}
$$

and $\left|Q_{y}\right|=\int_{Q_{y}} d o$. Hence integration has been replaced by a quadrature formula (midpoint rule). Thus considering (4.1) on the collocation grid only, we obtain a linear algebraic system for $3(2 L+1)^{2}$ unknowns $\left(3\right.$ components, $(2 L+1)^{2}$ collocation points) with a non-sparse but diagonal-dominant system matrix, which is invertible for sufficiently small $\sigma>0$. This follows by the usual perturbation theory from the fact that (3.28) is uniquely solvable in $C(S)^{3}$. Moreover, the following estimates can be obtained in the case of boundary values $b \in C(S)^{3}$ as in $[6]$ :

$$
\begin{array}{r}
\max _{x \in S}\left|\Psi(x)-\Psi_{\sigma}(x)\right| \leq c(\lambda) \sigma \ln (1 / \sigma), \\
\max _{x \in G_{\sigma}}\left|D_{\lambda}^{*} \Psi(x)-D_{\lambda, \sigma}^{*} \Psi_{\sigma}(x)\right| \leq c(\lambda) \sigma \ln (1 / \sigma) .
\end{array}
$$

Here $\left(G_{\sigma}\right)_{\sigma>0}$ is a family of subregions $G_{\sigma}$ exhausting $G$ as $\sigma \rightarrow 0$.

Extending both grids from the boundary $S$ into the domain $G$, the volume potentials can be approximated analogously, using the midpoint rule as quadrature formula instead of integration. In the following, we present some test calculations for the non-stationary Stokes equations (1.1) in the 3D unit ball, which have been performed without using any symmetry property of the ball: Let

$$
\begin{aligned}
& (t, x) \rightarrow v(t, x)=(t+1)\left(\exp \left(-r^{2}\right)-\exp (-1)\right)\left(\begin{array}{l}
x_{3}-x_{2} \\
x_{1}-x_{3} \\
x_{1}-x_{2}
\end{array}\right), \\
& (t, x) \rightarrow p(t, x)=\text { constant }, \quad r=\left(x_{1}^{2}+x_{2}^{2}+x_{3}^{2}\right)^{1 / 2} .
\end{aligned}
$$

Then $v, \nabla p$ is the unique solution of a constructed non-stationary Stokes problem 
(1.1) with

$$
\nu=1, \quad F=D_{t} v-\Delta v+\nabla p, \quad v_{0}=v(0) .
$$

The following numerical results give some idea about the accuracy of our approach. They have been obtained for the time step size $h=0.1$ and the spatial step size $\sigma=1 / 16$ with single precision. Let $E(j), j=1,2,3$, denote the mean (in space) relative error (\%), i.e.

$$
E(j)=\frac{100}{L} \sum_{l=1}^{L}\left|\frac{v_{j}^{\mathrm{appr}}\left(x_{l}\right)-v_{j}^{\text {exa }}\left(x_{l}\right)}{v_{j}^{\text {exa }}\left(x_{l}\right)}\right| .
$$

Development in time of $E(j)$

\begin{tabular}{|c|c|c|c|}
\hline$t$ & $j=1$ & $j=2$ & $j=3$ \\
\hline 0.1 & 1.215 & 1.594 & 2.932 \\
0.2 & 1.316 & 1.373 & 2.721 \\
0.3 & 1.517 & 1.354 & 2.490 \\
0.4 & 1.615 & 1.354 & 2.331 \\
0.5 & 1.748 & 1.392 & 2.229 \\
0.6 & 1.872 & 1.444 & 2.159 \\
0.7 & 1.931 & 1.454 & 2.099 \\
0.8 & 1.991 & 1.466 & 2.049 \\
0.9 & 2.049 & 1.478 & 2.012 \\
1.0 & 2.108 & 1.492 & 1.985 \\
\hline
\end{tabular}

\section{References}

[1] W. Borchers, Über das Anfangsrandwertproblem der instationären Stokes Gleichung, Z. Angew. Math. Mech. 65 (1985), T329-T330.

[2] L. Cattabriga, Su un problema al contorno relativo al sistema di equazioni di Stokes, Sem. Mat. Univ. Padova 31 (1964), 308-340.

[3] M. Costabel, Principles of boundary element methods, preprint 998, Technische Hochschule Darmstadt, 1986.

[4] P. Deuring, W. von Wahl and P. Weidemaier, Das lineare Stokes-System im $\mathbb{R}^{3}$ (1. Vorlesungen über das Innenraumproblem), Bayreuth. Math. Schr. 27 (1988), 1-252.

[5] H. Fujita and T. Kato, On the Navier-Stokes initial value problem I, Arch. Rational Mech. Anal. 16 (1964), 269-315.

[6] F. K. Hebeker, Efficient boundary element methods for three-dimensional exterior viscous flow, Numer. Methods Partial Differential Equations 2 (1986), 273-297.

[7] G. C. Hsiao, P. Kopp and W. L. Wendland, A Galerkin collocation method for some integral equations of the first kind, Computing 25 (1980), 89-130.

[8] - - - - Some applications of a Galerkin-collocation method for boundary integral equations of the first kind, Math. Methods Appl. Sci. 6 (1984), 280-325.

[9] J. Kačur, Method of Rothe in Evolution Equations, Teubner-Texte Math. 80, Teubner Leipzig 1985.

[10] O. A. Ladyzhenskaya, The Mathematical Theory of Viscous Incompressible Flow, Gordon and Breach, New York 1969.

[11] P. D. Lax and R. D. Richtmyer, Survey of the stability of linear finite difference equations, Comm. Pure Appl. Math. 9 (1956), 267-293. 
[12] R. Leis, Vorlesungen über partielle Differentialgleichungen zweiter Ordnung, Bibliographisches Institut, Mannheim 1967.

[13] M. McCracken, The resolvent problem for the Stokes equations on halfspace in $L_{p}$, SIAM J. Math. Anal. 12 (1981), 201-228.

[14] F. K. G. Odquist, Über die Randwertaufgaben der Hydrodynamik zäher Flüssigkeiten, Math. Z. 32 (1930), 329-375.

[15] W. I. Smirnow, Lehrgang der höheren Mathematik 4, Deutscher Verlag der Wissenschaften, Berlin 1979.

[16] R. Temam, Navier-Stokes Equations, North-Holland, Amsterdam 1977.

[17] W. Varnhorn, Efficient quadrature for a boundary element method to compute threedimensional Stokes flow, Internat. J. Numer. Methods Fluids 9 (1989), 185-191. 